\title{
First branching fraction measurement of the suppressed decay $\Xi_{c}^{0} \rightarrow \pi^{-} \Lambda_{c}^{+}$
}

\author{
R. Aaij et al.* \\ (LHCb Collaboration)
}

(Received 23 July 2020; accepted 11 September 2020; published 12 October 2020)

\begin{abstract}
The $\Xi_{c}^{0}$ baryon is unstable and usually decays into charmless final states by the $c \rightarrow s u \bar{d}$ transition. It can, however, also disintegrate into a $\pi^{-}$meson and a $\Lambda_{c}^{+}$baryon via $s$ quark decay or via $c s \rightarrow d c$ weak scattering. The interplay between the latter two processes governs the size of the branching fraction $\mathcal{B}\left(\Xi_{c}^{0} \rightarrow \pi^{-} \Lambda_{c}^{+}\right)$, first measured here to be $(0.55 \pm 0.02 \pm 0.18) \%$, where the first uncertainty is statistical and second systematic. This result is compatible with the larger of the theoretical predictions that connect models of hyperon decays using partially conserved axial currents and SU(3) symmetry with those involving the heavy-quark expansion and heavy-quark symmetry. In addition, the branching fraction of the normalization channel, $\mathcal{B}\left(\Xi_{c}^{+} \rightarrow p K^{-} \pi^{+}\right)=(1.135 \pm 0.002 \pm 0.387) \%$ is measured.
\end{abstract}

DOI: 10.1103/PhysRevD.102.071101

Baryons containing both an $s$ quark and a heavy $c$ or $b$ quark, denoted as $Q$, usually decay via the disintegration of the heavy quark. There is, however, the possibility of $s$ quark decay causing the transformation. Theoretical predictions concerning the decay widths of $\Xi_{Q} \rightarrow \pi \Lambda_{Q}$ transitions are based on the size of the $s$ quark decay amplitude $s \rightarrow u(\bar{u} d)$ (SUUD) and the weak scattering (WS) amplitude $Q s \rightarrow d Q$ [1]. Feynman diagrams corresponding to these amplitudes are shown in Fig. 1 for $\Xi_{c}^{0}$ decay.

Studies of these $\Xi_{Q}$ baryon decays provide a connection to theories concerning hyperon decays with those for the heavy $b$ and $c$ quarks. The former use partially conserved axial currents (PCAC) and SU(3) symmetry [2], whereas the latter apply more modern approaches using four-quark operators, including the heavy quark expansion, and heavyquark symmetry (HQS). As the $\Xi_{b}^{-}$baryon consists of $b, s$, and $d$ quarks, the WS amplitude is not present in $\Xi_{b}^{-} \rightarrow$ $\pi^{-} \Lambda_{b}^{0}$ decays, so the measurement of that decay rate can be used to determine the SUUD amplitude. This information can be used to predict the $\Xi_{c}^{0}$ decay rate that, in principle, involves both amplitudes. Whenever a specific final state is mentioned additional use of the charge-conjugated state is implied.

The well-known $\Xi_{c}^{0}$ baryon consists of the $c, s$, and $d$ quarks, and has a lifetime of $154.5 \pm 1.7 \pm 1.6 \pm 1.0 \mathrm{fs}$ [3]. The branching fraction $\mathcal{B}\left(\Xi_{c}^{0} \rightarrow \pi^{-} \Lambda_{c}^{+}\right)$has not been previously measured. Several authors have made predictions using the measured SUUD amplitude and the

*Full author list given at the end of the article.

Published by the American Physical Society under the terms of the Creative Commons Attribution 4.0 International license. Further distribution of this work must maintain attribution to the author(s) and the published article's title, journal citation, and DOI. Funded by SCOAP ${ }^{3}$. measured lifetimes of the $\mathrm{SU}(3)$ triplet baryons $\Xi_{c}^{0}, \Lambda_{c}^{+}$, and $\Xi_{c}^{+}$, as input for determining the WS amplitude. This method was pioneered by Voloshin [1] where he used SU(3) symmetry, PCAC and the heavy-quark limit to determine an upper limit on $\Gamma\left(\Xi_{b}^{-} \rightarrow \pi^{-} \Lambda_{b}^{0}\right)$. In a subsequent paper, he uses the input from the LHCb measurement of $\mathcal{B}\left(\Xi_{b}^{-} \rightarrow \pi^{-} \Lambda_{b}^{0}\right)=(0.60 \pm 0.18) \%$ [4] and updated values for the charmed baryon lifetimes to find the SUUD rate and then calculates the WS amplitude. He predicts $\mathcal{B}\left(\Xi_{c}^{0} \rightarrow \pi^{-} \Lambda_{c}^{+}\right) \gtrsim(0.25 \pm 0.15) \times 10^{-3}$ [5], assuming negative interference between the two strangeness-changing amplitudes.

Gronau and Rosner, using the same approach as Voloshin, predict two possible branching fractions for $\Xi_{c}^{0} \rightarrow \pi^{-} \Lambda_{c}^{+}$decay, depending on the sign of the interference between the two decay amplitudes [6]. Based on the measured $\mathcal{B}\left(\Xi_{b}^{-} \rightarrow \pi^{-} \Lambda_{b}^{0}\right)$ [4], and using charmed-baryon lifetimes available at that time, they predict $\mathcal{B}\left(\Xi_{c}^{0} \rightarrow\right.$ $\left.\pi^{-} \Lambda_{c}^{+}\right)=(0.19 \pm 0.07) \%$ for constructive interference and $\mathcal{B}\left(\Xi_{c}^{0} \rightarrow \pi^{-} \Lambda_{c}^{+}\right) \lesssim 0.01 \%$ for destructive interference between the SUUD and WS contributions. We have redone their calculation using updated lifetime measurements $[3,7]$, finding $\mathcal{B}\left(\Xi_{c}^{0} \rightarrow \pi^{-} \Lambda_{c}^{+}\right)=(0.14 \pm 0.07) \%$ for constructive interference and $\mathcal{B}\left(\Xi_{c}^{0} \rightarrow \pi^{-} \Lambda_{c}^{+}\right) \lesssim(0.018 \pm$ $0.015) \%$ for destructive interference. Faller and Mannel, on the other hand, predict $\mathcal{B}\left(\Xi_{c}^{0} \rightarrow \pi^{-} \Lambda_{c}^{+}\right)<0.3 \%$, an upper limit obtained by assuming constructive interference [8]. Finally, Cheng et al. predict $\mathcal{B}\left(\Xi_{c}^{0} \rightarrow \pi^{-} \Lambda_{c}^{+}\right) \sim 0.0087 \%$, assuming negative interference [9]. We have not updated these last predictions; the effect would be to lower Faller and Mannel's positive interference prediction and raise the Cheng et al. negative one, giving somewhat better agreement with Gronau and Rosner's predictions.

In this paper we measure $\mathcal{B}\left(\Xi_{c}^{0} \rightarrow \pi^{-} \Lambda_{c}^{+}\right)$using data collected by the LHCb detector, corresponding to $3.8 \mathrm{fb}^{-1}$ 

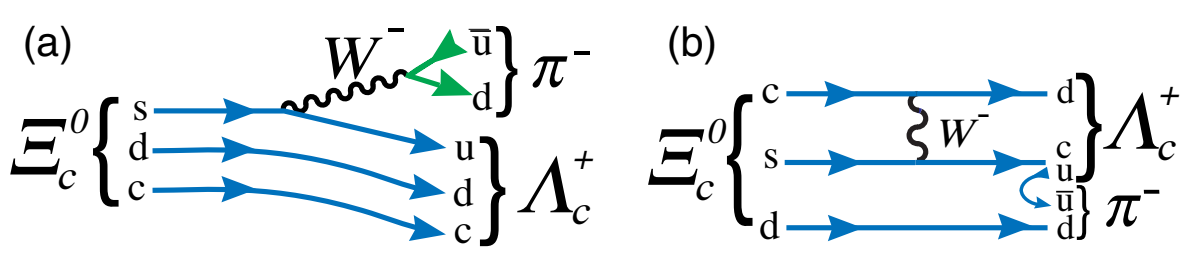

FIG. 1. Decay diagrams for $\Xi_{c}^{0} \rightarrow \pi^{-} \Lambda_{c}^{+}$transitions. (a) The SUUD amplitude, and (b) the WS amplitude.

of integrated luminosity in $13 \mathrm{TeV}$ center-of-mass energy $p p$ collisions taken in 2017 and 2018. Natural units are used in this paper with $c=\hbar=1$. The LHCb detector is a single-arm forward spectrometer covering the pseudorapidity range $2<\eta<5$, described in detail in Refs. [10,11]. The trigger [12] consists of a hardware stage, based on information from the calorimeter and muon systems, followed by a software stage, which reconstructs charged particles.

Simulation is required to model the effects of the detector acceptance and selection requirements. We generate $p p$ collisions using PYTHIA [13] with a specific LHCb configuration [14]. Decays of unstable particles are described by EVTGEN [15], where final-state radiation is generated using PнотоS [16]. The interaction of the particles with the detector, and its response, are implemented using the GEANT4 toolkit [17] as described in Ref. [18].

In our analysis we use the prompt $\Xi_{c}^{0}$ sample, i.e., baryons, and their excitations, produced directly in the $p p$ collisions. Measurement of $\mathcal{B}\left(\Xi_{c}^{0} \rightarrow \pi^{-} \Lambda_{c}^{+}\right)$is hampered by the lack of accurately measured $\Xi_{c}^{0}$ branching fractions [7] to be used for normalization. A measurement of $\mathcal{B}\left(\Xi_{c}^{0} \rightarrow\right.$ $\pi^{+} \Xi^{-}$) with a $29 \%$ uncertainty exists [19], but the efficiency for reconstructing $\Xi^{-}$baryons is low in $\mathrm{LHCb}$, in particular without a dedicated trigger line, so using this mode would lead to an unacceptably large error. We overcome this difficulty by using two indirect methods, described below, that require additional measurements of prompt $\Lambda_{c}^{+}$and $\Xi_{c}^{+}$yields, both reconstructed in the $p K^{-} \pi^{+}$ decay mode. The same decay mode is also used to reconstruct $\Lambda_{c}^{+}$from the $\Xi_{c}^{0} \rightarrow \pi^{-} \Lambda_{c}^{+}$decays.

We use a two-step process to maximize the statistical significance of our signal channel, as well as the two normalization channels. First, we apply a set of loose selection criteria to obtain samples with large signal efficiencies and suppressed background. Subsequently, we use three different boosted decision trees (BDT) $[20,21]$, one for each baryon decay, implemented in the TMVA toolkit [22], to further separate signal from background.

The loose selection criteria for the $p K^{-} \pi^{+}$final states include requirements on the tracks to have sufficient transverse momenta $\left(p_{\mathrm{T}}\right)$, be separated from the primary $p p$ collision vertex $(\mathrm{PV})$, form a three-track vertex, and be identified as the hypothesized particle species. For the $\Xi_{c}^{0} \rightarrow \pi^{-} \Lambda_{c}^{+}$decay we require, in addition, that the $p K^{-} \pi^{+}$ has a mass within $\pm 20 \mathrm{MeV}$ of the $\Lambda_{c}^{+}$mass peak; that there is an additional $\pi^{-}$meson, which when combined with the $\Lambda_{c}^{+}$candidate, has an invariant mass from $-85 \mathrm{MeV}$ below the known $\Xi_{c}^{0}$ mass [7] to $115 \mathrm{MeV}$ above; and that the $p_{\mathrm{T}}$ of the $\Xi_{c}^{0}$ candidate is greater than $5 \mathrm{GeV}$.

The BDTs are trained with background samples from data and simulated signal samples. Background training samples for the $\Lambda_{c}^{+}$and $\Xi_{c}^{+}$candidates are taken from the sideband regions on both sides of the mass peaks. For the $\Lambda_{c}^{+}$baryon background the intervals are $40-65 \mathrm{MeV}$ away from the known $\Lambda_{c}^{+}$mass [7]. For the $\Xi_{c}^{+}$baryon training the lower and higher sidebands are taken $40-58 \mathrm{MeV}$ and 40-72 MeV from the known $\Xi_{c}^{+}$mass [7], respectively. The $\Xi_{c}^{0}$ background is constructed from like-sign $\pi^{+} \Lambda_{c}^{+}$candidates within $\pm 5 \mathrm{MeV}$ of the known $\Xi_{c}^{0}$ baryon mass [7]. For the $\Lambda_{c}^{+}$and $\Xi_{c}^{+}$candidates, we compute the $p K^{-} \pi^{+}$ invariant mass after constraining the three decay particles to form a common vertex and the summed momentum vector to point to the PV; this fitter is referred to as the "decay tree fitter" (DTF) [23]. In the case of the $\Xi_{c}^{0}$ baryon we add the additional $\pi^{\mp}$ meson before performing the fit. Only $1 / 10$ of the available $\Lambda_{c}^{+} \rightarrow p K^{-} \pi^{+}$data sample is used to measure the $\Lambda_{c}^{+}$yield due to the large samples available relative to the other channels.

The variables used in the $\Lambda_{c}^{+}$and $\Xi_{c}^{+}$BDTs are the particle identification probabilities; the $\chi_{\mathrm{IP}}^{2}$ of the $p K^{-} \pi^{+}$ with respect to the primary vertex, where $\chi_{\mathrm{IP}}^{2}$ is defined as the difference in the vertex fit $\chi^{2}$ with and without the $p$, $K^{-}$, and $\pi^{+}$tracks; the angle between the particle's momentum vector and the vector from the original PV before the DTF refitting to the particle's decay vertex; the decay distance from the PV, and the DTF $\chi^{2}$. The $\Xi_{c}^{0}$ candidates are selected by a separate BDT using the same criteria used for the $\Lambda_{c}^{+}$by adding similar extra variables associated with the additional pion.

The BDT selections are optimized by maximizing the ratio of signal efficiency to the square root of the number of candidates in the regions where we expect signal peaks. We show the resulting mass spectra in Fig. 2; the data are fitted using the signal and background shapes described in the figure caption. The fit yields are $6320 \pm 230 \Xi_{c}^{0}$, $2667200 \pm 3300 \Lambda_{c}^{+}$, and $1613000 \pm 3500 \Xi_{c}^{+}$signal decays. To take into account the efficiency variation we perform the fits in four bins, two in $p_{\mathrm{T}}$ and two in $\eta$, and apply efficiencies calculated in each bin. 

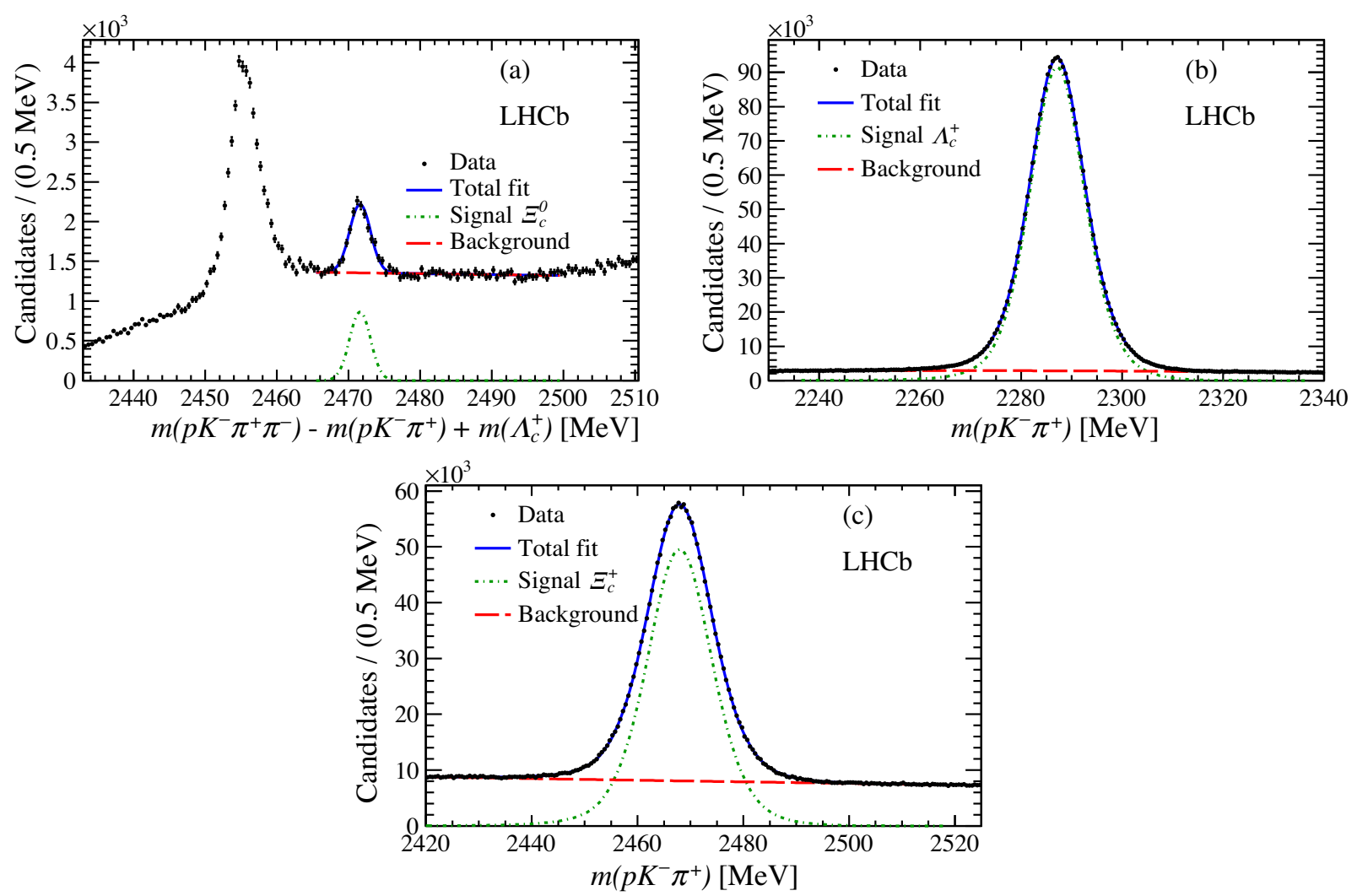

FIG. 2. Reconstructed invariant-mass distributions and signal fits of (a) $m\left(p K^{-} \pi^{+} \pi^{-}\right)$showing a large $\Sigma_{c}^{0}$ signal with a smaller $\Xi_{c}^{0}$ signal, (b) $m\left(p K^{-} \pi^{+}\right)$showing the $\Lambda_{c}^{+}$signal, and (c) $m\left(p K^{-} \pi^{+}\right)$showing the $\Xi_{c}^{+}$signal. For (a) the signal shape is a Crystal Ball function [24] with a high-mass tail, and the background shape is linear. For (b) and (c) the signal shapes are double-sided Crystal Ball plus single Gaussian functions, while the background shapes are second-order polynomials. The data in (b) only use $1 / 10$ of the available sample.

Trigger efficiencies are estimated from data, using the technique described in Ref. [25]. Selection efficiencies are determined using simulated events, which are weighted to reproduce the resonance structures in the $p K^{-} \pi^{+}$final states visible in the $\Lambda_{c}^{+}$and $\Xi_{c}^{+}$signal samples. The overall detection efficiencies are $(0.11 \pm 0.02) \%$, $[(0.35 \pm 0.01) / 10] \%$, and $(1.18 \pm 0.03) \%$ for $\Xi_{c}^{0}, \Lambda_{c}^{+}$, and $\Xi_{c}^{+}$decays, respectively, where the factor of 10 is the prescale.

The first normalization method uses the LHCb measurement of the relative production fractions of the $\Xi_{b}^{-}$and $\Lambda_{b}^{0}$ beauty baryons, $f_{\Xi_{b}^{-}} / f_{\Lambda_{b}^{0}}=(8.2 \pm 0.7 \pm 2.6) \%$ [26]. Using HQS we equate the unmeasured production ratio of $\Xi_{c}^{0}$ to $\Lambda_{c}^{+}$baryons, $f_{\Xi_{c}^{0}} / f_{\Lambda_{c}^{+}}$, to $\mathcal{C} \cdot f_{\Xi_{b}^{-}} / f_{\Lambda_{b}^{0}}$, where $\mathcal{C}$ is a correction factor for feed-downs of excited $\Xi_{b}$ baryons that do not have equal rates to $\Xi_{b}^{-}$and $\Xi_{b}^{0}$ final states. This feeddown is not symmetric primarily because the $\Xi_{b}^{\prime}(5935)^{0}$ state always decays to $\pi^{0}$ (or $\gamma$ ) $\Xi_{b}^{0}$ [27], since its mass is too low to decay into $\pi^{+} \Xi_{b}^{-}$. On the other hand, both the $\Xi_{b}^{\prime-}$ and $\Xi_{b}^{*-}$ states are seen to decay into both $\pi^{-} \Xi_{b}^{0}$ and $\pi^{0} \Xi_{b}^{-}$ final states [28]. Any not yet observed higher mass states would be isospin symmetric in their decays. Accounting for all the known excited states, and the associated phase-space corrections, results in $\mathcal{C}=1.18 \pm 0.04$, where the uncertainty arises from the errors on the relative branching fraction measurements.

The second method uses the recent Belle measurement $\mathcal{B}\left(\Xi_{c}^{+} \rightarrow p K^{-} \pi^{+}\right)=(0.45 \pm 0.21 \pm 0.07) \%$ [29]. Here we take the production of $\Xi_{c}^{0}$ baryons equal to that of $\Xi_{c}^{+}$by isospin symmetry, e.g., $f_{\Xi_{c}^{0}} / f_{\Xi_{c}^{+}}=1.00 \pm 0.01$ [30]. As the final state particles in the $\Xi_{c}^{+}$decay are the same as in the $\Lambda_{c}^{+}$decay, many systematic uncertainties cancel.

We determine $\mathcal{B}\left(\Xi_{c}^{0} \rightarrow \pi^{-} \Lambda_{c}^{+}\right)$using the two measured ratios

$$
\begin{aligned}
\mathcal{R}_{1} & \equiv \frac{N\left(\Xi_{c}^{0}\right)}{N\left(\Lambda_{c}^{+}\right)}=\frac{f_{\Xi_{c}^{0}}}{f_{\Lambda_{c}^{+}}} \cdot \mathcal{B}\left(\Xi_{c}^{0} \rightarrow \pi^{-} \Lambda_{c}^{+}\right) \\
& =(0.095 \pm 0.003 \pm 0.012) \%, \\
\mathcal{R}_{2} & \equiv \frac{N\left(\Xi_{c}^{0}\right)}{N\left(\Xi_{c}^{+}\right)}=\frac{f_{\Xi_{c}^{0}}}{f_{\Xi_{c}^{+}}} \cdot \frac{\mathcal{B}\left(\Lambda_{c}^{+} \rightarrow p K^{-} \pi^{+}\right)}{\mathcal{B}\left(\Xi_{c}^{+} \rightarrow p K^{-} \pi^{+}\right)} \cdot \mathcal{B}\left(\Xi_{c}^{0} \rightarrow \pi^{-} \Lambda_{c}^{+}\right) \\
& =(5.70 \pm 0.19 \pm 0.77) \%,
\end{aligned}
$$

where $N(i)$ indicates the efficiency corrected number of signal events for baryon $i, f_{i}$ indicates the fraction of particle production with respect to all $c$ - or $b$-quark 
production, and the uncertainties are statistical and systematic, respectively, a convention used in the rest of this paper. As discussed above, $f_{\Xi_{c}^{0}} / f_{\Lambda_{c}^{+}}=\mathcal{C} \cdot f_{\Xi_{b}^{-}} / f_{\Lambda_{b}^{0}}=$ $(9.7 \pm 0.9 \pm 3.1) \%$, where we have added a $5 \%$ relative systematic uncertainty, explained later, to account for our assumption of HQS.

We also determine $\mathcal{B}\left(\Xi_{c}^{+} \rightarrow p K^{-} \pi^{+}\right)$using

$$
\begin{aligned}
\mathcal{R}_{3} & \equiv \frac{N\left(\Xi_{c}^{+}\right)}{N\left(\Lambda_{c}^{+}\right)}=\frac{f_{\Xi_{c}^{+}}}{f_{\Lambda_{c}^{+}}} \cdot \frac{\mathcal{B}\left(\Xi_{c}^{+} \rightarrow p K^{-} \pi^{+}\right)}{\mathcal{B}\left(\Lambda_{c}^{+} \rightarrow p K^{-} \pi^{+}\right)} \\
& =(1.753 \pm 0.003 \pm 0.107) \%,
\end{aligned}
$$

where $\mathcal{B}\left(\Lambda_{c}^{+} \rightarrow p K^{-} \pi^{+}\right)=(6.23 \pm 0.33) \%$ [7]. The correlation matrix for these three results is

$$
\left(\begin{array}{cccc} 
& \mathcal{R}_{1} & \mathcal{R}_{2} & \mathcal{R}_{3} \\
\mathcal{R}_{1} & 1 & 0.71 & 0.15 \\
\mathcal{R}_{2} & \ldots & 1 & -0.18 \\
\mathcal{R}_{3} & \ldots & \ldots & 1
\end{array}\right)
$$

The derived branching fractions are

$$
\begin{aligned}
& \mathcal{B}_{1} \equiv \mathcal{B}\left(\Xi_{c}^{0} \rightarrow \pi^{-} \Lambda_{c}^{+}\right)=(0.98 \pm 0.04 \pm 0.35) \%, \\
& \mathcal{B}_{2} \equiv \mathcal{B}\left(\Xi_{c}^{0} \rightarrow \pi^{-} \Lambda_{c}^{+}\right)=(0.41 \pm 0.01 \pm 0.21) \%, \\
& \mathcal{B}_{3} \equiv \mathcal{B}\left(\Xi_{c}^{+} \rightarrow p K^{-} \pi^{+}\right)=(1.135 \pm 0.002 \pm 0.387) \% .
\end{aligned}
$$

Their correlation matrix is

$$
\left(\begin{array}{cccc} 
& \mathcal{B}_{1} & \mathcal{B}_{2} & \mathcal{B}_{3} \\
\mathcal{B}_{1} & 1 & 0.07 & 0.92 \\
\mathcal{B}_{2} & \ldots & 1 & -0.02 \\
\mathcal{B}_{3} & \ldots & \ldots & 1
\end{array}\right) .
$$

The weighted average value of $\mathcal{B}_{1}$ and $\mathcal{B}_{2}$, taking into account their correlated error, is

$$
\mathcal{B}\left(\Xi_{c}^{0} \rightarrow \pi^{-} \Lambda_{c}^{+}\right)=(0.55 \pm 0.02 \pm 0.18) \% .
$$

Systematic uncertainties dominate these results due to our reliance on external inputs. Our assumption of HQS to relate $f_{\Xi_{c}^{0}} / f_{\Lambda_{c}^{+}}$to $f_{\Xi_{b}^{-}} / f_{\Lambda_{b}^{0}}$ is justified by considering the analogous ratios of production fractions between charm and beauty states in $13 \mathrm{TeV} p p$ collisions, $\frac{f_{D_{s}^{+}}}{f_{D^{0}}+f_{D^{+}}}$and $\frac{f_{B_{s}^{0}}}{f_{B^{0}}+f_{B^{+}}}$. The beauty ratio is measured using semimuonic decays into a charmed meson, determined in the kinematic range $4<p_{\mathrm{T}}<25 \mathrm{GeV}$, and is equal to $0.122 \pm 0.006$ [31]. Using the total charm cross sections reported for $0<p_{\mathrm{T}}<15 \mathrm{GeV}$ in Ref. [32], we find $\frac{f_{D_{s}^{+}}}{f_{D^{0}}+f_{D^{+}}} \approx 0.121$, where the statistical uncertainty is negligible. The systematic uncertainties in the charm-meson ratio including tracking, particle identification, luminosity, etc., mostly cancel. The uncertainties in the charm meson branching fractions cancel in the comparison with the $B$ meson ratio, because the same values are used in both. Thus we are left with a few percent uncertainty in the comparison of the charm and beauty meson ratios. The $p_{\mathrm{T}}$ distributions of the ratios are somewhat different; they fall linearly in the beauty case [31] and are flatter in the charm case [32]. Taking this into account, a 5\% relative uncertainty due to the HQS assumption appears reasonable. Contamination of the charm baryons from $b$-decay sources is estimated in simulation and subtracted. The resultant systematic uncertainties in the ratios are small. Table I summarizes the sources of systematic uncertainty.

In conclusion, we perform the first measurement of the branching fraction of the suppressed $\Xi_{c}^{0} \rightarrow \pi^{-} \Lambda_{c}^{+}$decays, giving $\mathcal{B}\left(\Xi_{c}^{0} \rightarrow \pi^{-} \Lambda_{c}^{+}\right)=(0.55 \pm 0.02 \pm 0.18) \%$. We compare with the theoretical predictions in Fig. 3; while our measurements are somewhat larger, we are in agreement with Gronau and Rosner's constructive interference prediction. Our result is also consistent with the Faller and Mannel upper limit arrived at by assuming constructive interference [8]. We

TABLE I. Systematic uncertainties in the branching fraction measurements. Ghost tracks refers to uncertainties from falsely reconstructed tracks. PID refers to particle identification efficiencies. Intermediate decays refers to the uncertainties caused by inexact modeling of the resonant structures in the charmedbaryon decays. The $b$-decay sources refer to charmed baryons originating from $b$-baryon decays included in our primarily prompt samples. Relative $\int \mathcal{L}$ refers to minor differences in the accumulated luminosities of the data samples for each of the three decays. The summed uncertainties are obtained by adding the individual components in quadrature.

\begin{tabular}{lrcc}
\hline \hline & \multicolumn{3}{c}{ Estimate $(\%)$} \\
\cline { 2 - 4 } & $\mathcal{B}\left(\Xi_{c}^{0} \rightarrow \pi^{-} \Lambda_{c}^{+}\right)$ & $\mathcal{B}\left(\Xi_{c}^{+} \rightarrow p K^{-} \pi^{+}\right)$ \\
\cline { 2 - 4 } Source & $\mathcal{B}_{1}$ & $\mathcal{B}_{2}$ & $\mathcal{B}_{3}$ \\
\hline$f_{\Xi_{b}^{-}} / f_{\Lambda_{b}^{0}}$ & 32 & $\ldots$ & 32 \\
$f_{\Xi_{c}^{0}} / f_{\Lambda_{c}^{+}}=\mathcal{C} \cdot f_{\Xi_{b}^{-}} / f_{\Lambda_{b}^{0}}$ & 6 & $\ldots$ & 6 \\
$f_{\Xi_{c}^{0}} / f_{\Xi_{c}^{+}}=1$ & $\ldots$ & 1 & 1 \\
$\mathcal{B}\left(\Xi_{c}^{+} \rightarrow p K^{-} \pi^{+}\right)$ & $\ldots$ & 49 & $\ldots$ \\
$\mathcal{B}\left(\Lambda_{c}^{+} \rightarrow p K^{-} \pi^{+}\right)$ & $\ldots$ & 5 & 5 \\
Simulation statistics & 4 & 3 & 2 \\
Trigger efficiency & 7 & 8 & 2 \\
Ghost tracks & 2 & 2 & 0 \\
PID & 1 & 1 & 1 \\
Tracking efficiencies & 2 & 2 & 0 \\
Fit yields & 6 & 6 & 3 \\
Intermediate decays & 2 & 2 & 2 \\
$b$-decay sources & 2 & 0 & 2 \\
Lifetimes & 3 & 3 & 2 \\
Relative $\int \mathcal{L}$ & $\ldots$ & 1 & 1 \\
Sum of external & 33 & 49 & 33 \\
Sum of intrinsic & 12 & 13 & 6 \\
Sum of all & 35 & 51 & 34 \\
\hline \hline
\end{tabular}




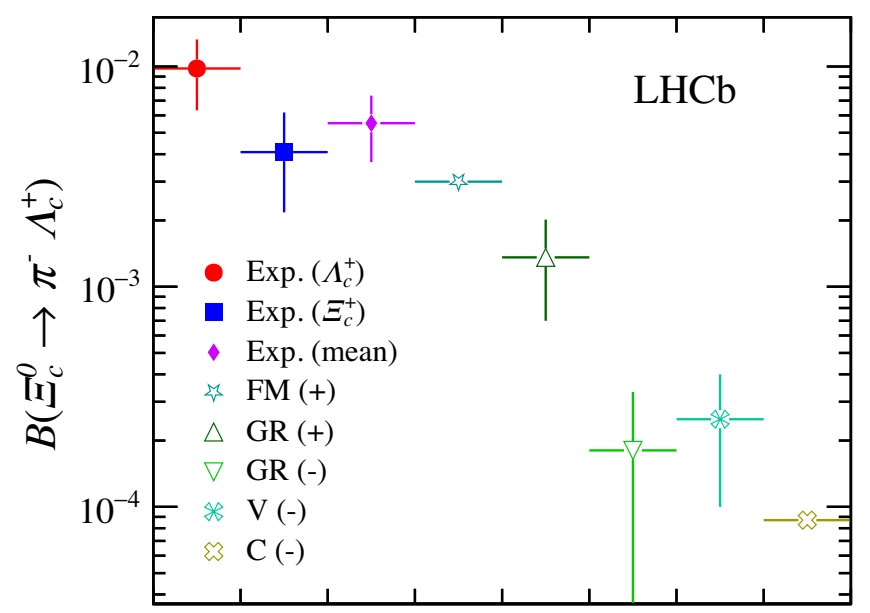

FIG. 3. Comparison of our two measurements of $\mathcal{B}\left(\Xi_{c}^{0} \rightarrow \pi^{-} \Lambda_{c}^{+}\right)$, and their average, with the lower limit of Voloshin (V) [5], the upper limit of Faller and Mannel [8] (FM), updated predictions of Gronau and Rosner [6] (GR), and Cheng et al. [9] (C). The (+ or -) indicates if positive or negative interference between the SUUD and WS amplitudes is assumed.

disagree, however, with Cheng's prediction of $\mathcal{B}\left(\Xi_{c}^{0} \rightarrow\right.$ $\pi^{-} \Lambda_{c}^{+}$) assuming negative interference [9]. In addition, the branching fraction of the normalization channel is found to be $\mathcal{B}\left(\Xi_{c}^{+} \rightarrow p K^{-} \pi^{+}\right)=(1.135 \pm 0.002 \pm 0.387) \%$, that is somewhat larger than, but in agreement with a previous Belle measurement [29], and has a better relative precision.
We express our gratitude to our colleagues in the CERN accelerator departments for the excellent performance of the LHC. We thank the technical and administrative staff at the LHCb institutes. We acknowledge support from CERN and from the national agencies: CAPES, CNPq, FAPERJ and FINEP (Brazil); MOST and NSFC (China); CNRS/ IN2P3 (France); BMBF, DFG and MPG (Germany); INFN (Italy); NWO (Netherlands); MNiSW and NCN (Poland); MEN/IFA (Romania); MSHE (Russia); MinECo (Spain); SNSF and SER (Switzerland); NASU (Ukraine); STFC (United Kingdom); DOE NP and NSF (USA). We acknowledge the computing resources that are provided by CERN, IN2P3 (France), KIT and DESY (Germany), INFN (Italy), SURF (Netherlands), PIC (Spain), GridPP (United Kingdom), RRCKI and Yandex LLC (Russia), CSCS (Switzerland), IFIN-HH (Romania), CBPF (Brazil), PL-GRID (Poland) and OSC (USA). We are indebted to the communities behind the multiple open-source software packages on which we depend. Individual groups or members have received support from AvH Foundation (Germany); EPLANET, Marie Skłodowska-Curie Actions and ERC (European Union); A*MIDEX, ANR, Labex P2IO and OCEVU, and Région Auvergne-Rhône-Alpes (France); Key Research Program of Frontier Sciences of CAS, CAS PIFI, and the Thousand Talents Program (China); RFBR, RSF and Yandex LLC (Russia); GVA, XuntaGal and GENCAT (Spain); the Royal Society and the Leverhulme Trust (United Kingdom).
[1] M. B. Voloshin, Weak decays $\Xi(Q) \rightarrow \Lambda(Q) \pi$, Phys. Lett. B 476, 297 (2000).

[2] S. Treiman, Commutator algebra and the PCAC hypothesis, Comments Nucl. Part. Phys. 1, 13 (1967).

[3] R. Aaij et al. (LHCb Collaboration), Precision measurement of the $\Lambda_{c}^{+}, \Xi_{c}^{+}$and $\Xi_{c}^{0}$ baryon lifetimes, Phys. Rev. D 100, 032001 (2019).

[4] R. Aaij et al. (LHCb Collaboration), Evidence for the Strangeness-Changing Weak Decay $\Xi_{b}^{-} \rightarrow \Lambda_{b}^{0} \pi^{-}$, Phys. Rev. Lett. 115, 241801 (2015).

[5] M. B. Voloshin, Update on splitting of lifetimes of $c$ and $b$ hyperons within the heavy quark expansion and decays $\Xi_{Q} \rightarrow \Lambda_{Q} \pi$, Phys. Rev. D 100, 114030 (2019).

[6] M. Gronau and J. L. Rosner, From $\Xi_{b} \rightarrow \Lambda_{b}^{0} \pi$ to $\Xi_{c} \rightarrow \Lambda_{c}^{+} \pi$, Phys. Lett. B 757, 330 (2016).

[7] M. Tanabashi et al. (Particle Data Group), Review of particle physics, Phys. Rev. D 98, 030001 (2018), and 2019 update.

[8] S. Faller and T. Mannel, Light-quark decays in heavy hadrons, Phys. Lett. B 750, 653 (2015).

[9] H.-Y. Cheng, C.-Y. Cheung, G.-L. Lin, Y.-C. Lin, T.-M. Yan, and H.-L. Yu, Heavy-flavor-conserving hadronic weak decays of heavy baryons, J. High Energy Phys. 03 (2016)
028; Heavy flavor conserving nonleptonic weak decays of heavy baryons, Phys. Rev. D 46, 5060 (1992).

[10] A. A. Alves, Jr.et al. (LHCb Collaboration), The LHCb detector at the LHC, J. Instrum. 3, S08005 (2008).

[11] R. Aaij et al. (LHCb Collaboration), LHCb detector performance, Int. J. Mod. Phys. A 30, 1530022 (2015).

[12] R. Aaij et al., The LHCb trigger and its performance in 2011, J. Instrum. 8, P04022 (2013).

[13] T. Sjöstrand, S. Mrenna, and P. Skands, A brief introduction to PYTHIA 8.1, Comput. Phys. Commun. 178, 852 (2008); PYTHIA 6.4 physics and manual, J. High Energy Phys. 05 (2006) 026.

[14] I. Belyaev et al., Handling of the generation of primary events in Gauss the LHCb simulation framework, J. Phys. Conf. Ser. 331, 032047 (2011).

[15] D. J. Lange, The EvtGen particle decay simulation package, Nucl. Instrum. Methods Phys. Res., Sect. A 462, 152 (2001).

[16] P. Golonka and Z. Was, PHOTOS Monte Carlo: A precision tool for QED corrections in $Z$ and $W$ decays, Eur. Phys. J. C 45, 97 (2006).

[17] J. Allison et al. (Geant4 Collaboration), Geant4 developments and applications, IEEE Trans. Nucl. Sci. 53, 270 
(2006); S. Agostinelli et al. (Geant4 Collaboration), Geant4: A simulation toolkit, Nucl. Instrum. Methods Phys. Res., Sect. A 506, 250 (2003).

[18] M. Clemencic, G. Corti, S. Easo, C. R. Jones, S. Miglioranzi, M. Pappagallo, and P. Robbe, The LHCb simulation application, Gauss: Design, evolution and experience, J. Phys. Conf. Ser. 331, 032023 (2011).

[19] Y. B. Li et al. (Belle Collaboration), First Measurements of Absolute Branching Fractions of the $\Xi_{c}^{0}$ Baryon at Belle, Phys. Rev. Lett. 122, 082001 (2019).

[20] L. Breiman, J.H. Friedman, R. A. Olshen, and C. J. Stone, Classification and Regression Trees (Wadsworth International Group, Belmont, CA, 1984).

[21] R. E. Schapire and Y. Freund, A decision-theoretic generalization of on-line learning and an application to boosting, J. Comput. Syst. Sci. 55, 119 (1997).

[22] A. Hoecker et al., TMVA: Toolkit for multivariate data analysis, Proc. Sci. ACAT2007 (2007) 040 [arXiv:physics/ 0703039]; J. Stelzer, A. Hocker, P. Speckmayer, and H. Voss, Current developments in TMVA: An outlook to TMVA4, Proc. Sci. ACAT08 (2008) 063.

[23] W. D. Hulsbergen, Decay chain fitting with a Kalman filter, Nucl. Instrum. Methods Phys. Res., Sect. A 552, 566 (2005).

[24] T. Skwarnicki, A study of the radiative cascade transitions between the Upsilon-prime and Upsilon resonances, Ph.D. thesis, Institute of Nuclear Physics, 1986, Report No. DESY-F31-86-02.
[25] S. Tolk, J. Albrecht, F. Dettori, and A. Pellegrino, Data driven trigger efficiency determination at LHCb, CERN Reports No. LHCb-PUB-2014-039 and No. CERN-LHCbPUB-2014-039, 2014.

[26] R. Aaij et al. (LHCb Collaboration), Measurement of the mass and production rate of $\Xi_{b}^{-}$baryons, Phys. Rev. D 99, 052006 (2019).

[27] R. Aaij et al. (LHCb Collaboration), Measurement of the properties of the $\Xi_{b}^{* 0}$ baryon, J. High Energy Phys. 05 (2016) 161.

[28] R. Aaij et al. (LHCb Collaboration), Observation of Two New $\Xi_{b}^{-}$Baryon Resonances, Phys. Rev. Lett. 114, 062004 (2015).

[29] Y. B. Li et al. (Belle Collaboration), First measurements of absolute branching fractions of the $\Xi_{c}^{+}$baryon at Belle, Phys. Rev. D 100, 031101 (2019).

[30] M. Tanabashi et al. (Particle Data Group), Review of particle physics, Phys. Rev. D 98, 030001 (2018), and 2019 update; see section 84.2.

[31] R. Aaij et al. (LHCb Collaboration), Measurement of $b$-hadron fractions in $13 \mathrm{TeV} p p$ collisions, Phys. Rev. D 100, 031102 (2019).

[32] R. Aaij et al. (LHCb Collaboration), Measurements of prompt charm production cross-sections in $p p$ collisions at $\sqrt{s}=13 \mathrm{TeV}$, J. High Energy Phys. 03 (2016) 159; Erratum, J. High Energy Phys. 09 (2016) 013(E); Erratum, J. High Energy Phys. 05 (2017) 074(E).

R. Aaij, ${ }^{31}$ C. Abellán Beteta, ${ }^{49}$ T. Ackernley, ${ }^{59}$ B. Adeva, ${ }^{45}$ M. Adinolfi, ${ }^{53}$ H. Afsharnia, ${ }^{9}$ C. A. Aidala, ${ }^{84}$ S. Aiola, ${ }^{25}$ Z. Ajaltouni, ${ }^{9}$ S. Akar, ${ }^{64}$ J. Albrecht, ${ }^{14}$ F. Alessio, ${ }^{47}$ M. Alexander, ${ }^{58}$ A. Alfonso Albero, ${ }^{44}$ Z. Aliouche, ${ }^{61}$ G. Alkhazov, ${ }^{37}$ P. Alvarez Cartelle, ${ }^{47}$ S. Amato, ${ }^{2}$ Y. Amhis, ${ }^{11}$ L. An, ${ }^{21}$ L. Anderlini, ${ }^{21}$ G. Andreassi, ${ }^{48}$ A. Andreianov,${ }^{37}$ M. Andreotti, ${ }^{20}$ F. Archilli, ${ }^{16}$ A. Artamonov, ${ }^{43}$ M. Artuso, ${ }^{67}$ K. Arzymatov,${ }^{41}$ E. Aslanides, ${ }^{10}$ M. Atzeni, ${ }^{49}$ B. Audurier, ${ }^{11}$ S. Bachmann, ${ }^{16}$ M. Bachmayer, ${ }^{48}$ J. J. Back ${ }^{55}$ S. Baker ${ }^{60}$ P. Baladron Rodriguez, ${ }^{45}$ V. Balagura,,${ }^{11, b}$ W. Baldini, ${ }^{20}$ J. Baptista Leite, ${ }^{1}$ R. J. Barlow ${ }^{61}$ S. Barsuk, ${ }^{11}$ W. Barter, ${ }^{60}$ M. Bartolini,${ }^{23,47, h}$ F. Baryshnikov, ${ }^{80}$ J. M. Basels, ${ }^{13}$ G. Bassi, ${ }^{28}$ V. Batozskaya,${ }^{35}$ B. Batsukh, ${ }^{67}$ A. Battig, ${ }^{14}$ A. Bay, ${ }^{48}$ M. Becker,${ }^{14}$ F. Bedeschi, ${ }^{28}$ I. Bediaga, ${ }^{1}$ A. Beiter,${ }^{67}$ V. Belavin,${ }^{41}$ S. Belin, ${ }^{26}$ V. Bellee ${ }^{48}$ K. Belous ${ }^{43}$ I. Belyaev, ${ }^{38}$ G. Bencivenni, ${ }^{22}$ E. Ben-Haim, ${ }^{12}$ A. Berezhnoy, ${ }^{39}$ R. Bernet, ${ }^{49}$ D. Berninghoff, ${ }^{16}$

H. C. Bernstein, ${ }^{67}$ C. Bertella, ${ }^{47}$ E. Bertholet, ${ }^{12}$ A. Bertolin, ${ }^{27}$ C. Betancourt,${ }^{49}$ F. Betti, ${ }^{19, \mathrm{e}}$ M. O. Bettler,${ }^{54}$ Ia. Bezshyiko, ${ }^{49}$ S. Bhasin, ${ }^{53}$ J. Bhom, ${ }^{33}$ L. Bian, ${ }^{72}$ M. S. Bieker, ${ }^{14}$ S. Bifani, ${ }^{52}$ P. Billoir, ${ }^{12}$ M. Birch, ${ }^{60}$ F. C. R. Bishop, ${ }^{54}$ A. Bizzeti, ${ }^{21, \mathrm{~s}}$ M. Bjørn, ${ }^{62}$ M. P. Blago, ${ }^{47}$ T. Blake,${ }^{55}$ F. Blanc, ${ }^{48}$ S. Blusk,${ }^{67}$ D. Bobulska, ${ }^{58}$ V. Bocci, ${ }^{30}$ J. A. Boelhauve, ${ }^{14}$ O. Boente Garcia, ${ }^{45}$ T. Boettcher, ${ }^{63}$ A. Boldyrev, ${ }^{81}$ A. Bondar, ${ }^{42, v}$ N. Bondar, ${ }^{37,47}$ S. Borghi, ${ }^{61}$ M. Borisyak, ${ }^{41}$ M. Borsato, ${ }^{16}$ J. T. Borsuk, ${ }^{33}$ S. A. Bouchiba, ${ }^{48}$ T. J. V. Bowcock, ${ }^{59}$ A. Boyer, ${ }^{47}$ C. Bozzi,${ }^{20}$ M. J. Bradley, ${ }^{60}$ S. Braun, ${ }^{65}$

A. Brea Rodriguez, ${ }^{45}$ M. Brodski, ${ }^{47}$ J. Brodzicka, ${ }^{33}$ A. Brossa Gonzalo, ${ }^{55}$ D. Brundu, ${ }^{26}$ A. Buonaura, ${ }^{49}$ C. Burr, ${ }^{47}$ A. Bursche, ${ }^{26}$ A. Butkevich, ${ }^{40}$ J. S. Butter ${ }^{31}$ J. Buytaert, ${ }^{47}$ W. Byczynski, ${ }^{47}$ S. Cadeddu, ${ }^{26}$ H. Cai, ${ }^{72}$ R. Calabrese, ${ }^{20, g}$ L. Calero Diaz, ${ }^{22}$ S. Cali, ${ }^{22}$ R. Calladine, ${ }^{52}$ M. Calvi, ${ }^{24, i}$ M. Calvo Gomez, ${ }^{83}$ P. Camargo Magalhaes, ${ }^{53}$ A. Camboni, ${ }^{44}$ P. Campana, ${ }^{22}$ D. H. Campora Perez ${ }^{47}$ A. F. Campoverde Quezada, ${ }^{5}$ S. Capelli, ${ }^{24, \mathrm{i}}$ L. Capriotti, ${ }^{19, \mathrm{e}}$ A. Carbone, ${ }^{19, \mathrm{e}}$ G. Carboni, ${ }^{29}$ R. Cardinale, ${ }^{23, h}$ A. Cardini, ${ }^{26}$ I. Carli, ${ }^{6}$ P. Carniti, ${ }^{24, i}$ K. Carvalho Akiba, ${ }^{31}$ A. Casais Vidal, ${ }^{45}$ G. Casse, ${ }^{59}$ M. Cattaneo, ${ }^{47}$ G. Cavallero, ${ }^{47}$ S. Celani, ${ }^{48}$ R. Cenci, ${ }^{28}$ J. Cerasoli, ${ }^{10}$ A. J. Chadwick, ${ }^{59}$ M. G. Chapman, ${ }^{53}$ M. Charles, ${ }^{12}$ Ph. Charpentier, ${ }^{47}$ G. Chatzikonstantinidis, ${ }^{52}$ M. Chefdeville, ${ }^{8}$ C. Chen, ${ }^{3}$ S. Chen, ${ }^{26}$ A. Chernov, ${ }^{33}$ S.-G. Chitic, ${ }^{47}$ V. Chobanova, ${ }^{45}$ S. Cholak, ${ }^{48}$ M. Chrzaszcz, ${ }^{33}$ A. Chubykin, ${ }^{37}$ V. Chulikov,${ }^{37}$ P. Ciambrone, ${ }^{22}$ M. F. Cicala,${ }^{55}$ X. Cid Vidal, ${ }^{45}$ G. Ciezarek, ${ }^{47}$ P. E. L. Clarke, ${ }^{57}$ M. Clemencic, ${ }^{47}$ H. V. Cliff, ${ }^{54}$ J. Closier, ${ }^{47}$ J. L. Cobbledick, ${ }^{61}$ V. Coco, ${ }^{47}$ J. A. B. Coelho, ${ }^{11}$ J. Cogan, ${ }^{10}$ E. Cogneras, ${ }^{9}$ L. Cojocariu, ${ }^{36}$ P. Collins, ${ }^{47}$ T. Colombo, ${ }^{47}$ A. Contu, ${ }^{26}$ N. Cooke, ${ }^{52}$ G. Coombs,${ }^{58}$ G. Corti, ${ }^{47}$ 
C. M. Costa Sobral,${ }^{55}$ B. Couturier, ${ }^{47}$ D. C. Craik,${ }^{63}$ J. Crkovská, ${ }^{66}$ M. Cruz Torres, ${ }^{1}$ R. Currie, ${ }^{57}$ C. L. Da Silva, ${ }^{66}$ E. Dall'Occo, ${ }^{14}$ J. Dalseno, ${ }^{45}$ C. D'Ambrosio, ${ }^{47}$ A. Danilina, ${ }^{38}$ P. d'Argent ${ }^{47}$ A. Davis,${ }^{61}$ O. De Aguiar Francisco,${ }^{47}$ K. De Bruyn ${ }^{77}$ S. De Capua ${ }^{61}$ M. De Cian, ${ }^{48}$ J. M. De Miranda, ${ }^{1}$ L. De Paula, ${ }^{2}$ M. De Serio, ${ }^{18, d}$ D. De Simone, ${ }^{49}$ P. De Simone, ${ }^{22}$ J. A. de Vries,${ }^{78}$ C. T. Dean, ${ }^{66}$ W. Dean,${ }^{84}$ D. Decamp,${ }^{8}$ L. Del Buono, ${ }^{12}$ B. Delaney, ${ }^{54}$ H.-P. Dembinski, ${ }^{14}$ A. Dendek,${ }^{34}$ X. Denis ${ }^{72}$ V. Denysenko, ${ }^{49}$ D. Derkach,${ }^{81}$ O. Deschamps, ${ }^{9}$ F. Desse, ${ }^{11}$ F. Dettori, ${ }^{26, f}$ B. Dey,${ }^{72}$ P. Di Nezza, ${ }^{22}$ S. Didenko, ${ }^{80}$ H. Dijkstra, ${ }^{47}$ V. Dobishuk, ${ }^{51}$ A. M. Donohoe,${ }^{17}$ F. Dordei,${ }^{26}$ M. Dorigo, ${ }^{28, w}$ A. C. dos Reis, ${ }^{1}$ L. Douglas,${ }^{58}$ A. Dovbnya, ${ }^{50}$ A. G. Downes, ${ }^{8}$ K. Dreimanis,${ }^{59}$ M. W. Dudek, ${ }^{33}$ L. Dufour, ${ }^{47}$ V. Duk, ${ }^{76}$ P. Durante, ${ }^{47}$ J. M. Durham, ${ }^{66}$

D. Dutta, ${ }^{61}$ M. Dziewiecki, ${ }^{16}$ A. Dziurda, ${ }^{33}$ A. Dzyuba, ${ }^{37}$ S. Easo, ${ }^{56}$ U. Egede,${ }^{69}$ V. Egorychev, ${ }^{38}$ S. Eidelman, ${ }^{42, v}$ S. Eisenhardt, ${ }^{57}$ S. Ek-In, ${ }^{48}$ L. Eklund, ${ }^{58}$ S. Ely, ${ }^{67}$ A. Ene, ${ }^{36}$ E. Epple, ${ }^{66}$ S. Escher, ${ }^{13}$ J. Eschle, ${ }^{49}$ S. Esen, ${ }^{31}$ T. Evans, ${ }^{47}$ A. Falabella, ${ }^{19}$ J. Fan, ${ }^{3}$ Y. Fan, ${ }^{5}$ B. Fang, ${ }^{72}$ N. Farley, ${ }^{52}$ S. Farry, ${ }^{59}$ D. Fazzini, ${ }^{24, i}$ P. Fedin, ${ }^{38}$ M. Féo, ${ }^{47}$

P. Fernandez Declara, ${ }^{47}$ A. Fernandez Prieto, ${ }^{45}$ F. Ferrari, ${ }^{19, e}$ L. Ferreira Lopes ${ }^{48}$ F. Ferreira Rodrigues, ${ }^{2}$ S. Ferreres Sole ${ }^{31}$ M. Ferrillo, ${ }^{49}$ M. Ferro-Luzzi, ${ }^{47}$ S. Filippov, ${ }^{40}$ R. A. Fini, ${ }^{18}$ M. Fiorini, ${ }^{20, g}$ M. Firlej, ${ }^{34}$ K. M. Fischer,${ }^{62}$ C. Fitzpatrick, ${ }^{61}$ T. Fiutowski, ${ }^{34}$ F. Fleuret, ${ }^{11, b}$ M. Fontana,${ }^{47}$ F. Fontanelli, ${ }^{23, h}$ R. Forty,${ }^{47}$ V. Franco Lima,${ }^{59}$ M. Franco Sevilla, ${ }^{65}$ M. Frank,${ }^{47}$ E. Franzoso, ${ }^{20}$ G. Frau, ${ }^{16}$ C. Frei, ${ }^{47}$ D. A. Friday, ${ }^{58}$ J. Fu, ${ }^{25, o}$ Q. Fuehring, ${ }^{14}$ W. Funk, ${ }^{47}$ E. Gabriel, ${ }^{31}$ T. Gaintseva, ${ }^{41}$ A. Gallas Torreira, ${ }^{45}$ D. Galli, ${ }^{19, \mathrm{e}}$ S. Gallorini, ${ }^{27}$ S. Gambetta, ${ }^{57}$ Y. Gan, ${ }^{3}$ M. Gandelman, ${ }^{2}$ P. Gandini,${ }^{25}$ Y. Gao, ${ }^{4}$ M. Garau, ${ }^{26}$ L. M. Garcia Martin, ${ }^{46}$ P. Garcia Moreno, ${ }^{44}$ J. García Pardiñas, ${ }^{49}$ B. Garcia Plana, ${ }^{45}$ F. A. Garcia Rosales, ${ }^{11}$ L. Garrido, ${ }^{44}$ D. Gascon, ${ }^{44}$ C. Gaspar, ${ }^{47}$ R. E. Geertsema, ${ }^{31}$ D. Gerick, ${ }^{16}$ L. L. Gerken, ${ }^{14}$ E. Gersabeck, ${ }^{61}$ M. Gersabeck, ${ }^{61}$ T. Gershon, ${ }^{55}$ D. Gerstel, ${ }^{10} \mathrm{Ph}$. Ghez, ${ }^{8}$ V. Gibson, ${ }^{54}$ A. Gioventù,${ }^{45}$ P. Gironella Gironell,${ }^{44}$ L. Giubega,${ }^{36}$ C. Giugliano, ${ }^{20, g}$ K. Gizdov,${ }^{57}$ V. V. Gligorov, ${ }^{12}$ C. Göbel,${ }^{70}$ E. Golobardes,${ }^{83}$ D. Golubkov,${ }^{38}$ A. Golutvin, ${ }^{60,80}$ A. Gomes, ${ }^{1, a}$ S. Gomez Fernandez, ${ }^{44}$ M. Goncerz, ${ }^{33}$ P. Gorbounov, ${ }^{38}$ I. V. Gorelov, ${ }^{39}$ C. Gotti,${ }^{24}$ E. Govorkova, ${ }^{31}$ J. P. Grabowski, ${ }^{16}$ R. Graciani Diaz, ${ }^{44}$ T. Grammatico, ${ }^{12}$ L. A. Granado Cardoso, ${ }^{47}$ E. Graugés, ${ }^{44}$ E. Graverini, ${ }^{48}$ G. Graziani, ${ }^{21}$ A. Grecu, ${ }^{36}$ L. M. Greeven, ${ }^{31}$ P. Griffith, ${ }^{20}$ L. Grillo, ${ }^{61}$ L. Gruber ${ }^{47}$ B. R. Gruberg Cazon, ${ }^{62}$ C. Gu, ${ }^{3}$ M. Guarise, ${ }^{20}$ P. A. Günther, ${ }^{16}$ E. Gushchin, ${ }^{40}$ A. Guth, ${ }^{13}$ Yu. Guz, ${ }^{43,47}$ T. Gys, ${ }^{47}$ T. Hadavizadeh, ${ }^{69}$ G. Haefeli, ${ }^{48}$ C. Haen, ${ }^{47}$ S. C. Haines, ${ }^{54}$ P. M. Hamilton, ${ }^{65}$ Q. Han ${ }^{7}$ X. Han, ${ }^{16}$ T. H. Hancock, ${ }^{62}$ S. Hansmann-Menzemer, ${ }^{16}$ N. Harnew, ${ }^{62}$ T. Harrison, ${ }^{59}$ R. Hart, ${ }^{31}$ C. Hasse,${ }^{47}$ M. Hatch, ${ }^{47}$ J. He, ${ }^{5}$ M. Hecker, ${ }^{60}$ K. Heijhoff, ${ }^{31}$ K. Heinicke, ${ }^{14}$ A. M. Hennequin, ${ }^{47}$ K. Hennessy, ${ }^{59}$ L. Henry, ${ }^{25,46}$ J. Heuel, ${ }^{13}$ A. Hicheur, ${ }^{68}$ D. Hill,,${ }^{62}$ M. Hilton, ${ }^{61}$ S. E. Hollitt, ${ }^{14}$ P. H. Hopchev, ${ }^{48}$ J. Hu, ${ }^{16}$ J. Hu, ${ }^{71}$ W. Hu, W. Huang, ${ }^{5}$ W. Hulsbergen, ${ }^{31}$ R. J. Hunter, ${ }^{55}$ M. Hushchyn, ${ }^{81}$ D. Hutchcroft, ${ }^{59}$ D. Hynds, ${ }^{31}$ P. Ibis, ${ }^{14}$ M. Idzik,${ }^{34}$ D. Ilin, ${ }^{37}$ P. Ilten, ${ }^{52}$ A. Inglessi, ${ }^{37}$ K. Ivshin, ${ }^{37}$ R. Jacobsson, ${ }^{47}$ S. Jakobsen, ${ }^{47}$ E. Jans, ${ }^{31}$ B. K. Jashal, ${ }^{46}$ A. Jawahery, ${ }^{65}$ V. Jevtic, ${ }^{14}$ F. Jiang, ${ }^{3}$ M. John, ${ }^{62}$ D. Johnson, ${ }^{47}$ C. R. Jones, ${ }^{54}$ T. P. Jones, ${ }^{55}$ B. Jost, ${ }^{47}$ N. Jurik, ${ }^{47}$ S. Kandybei, ${ }^{50}$ Y. Kang, ${ }^{3}$ M. Karacson, ${ }^{47}$ J. M. Kariuki,${ }^{53}$ N. Kazeev, ${ }^{81}$ M. Kecke,${ }^{16}$ F. Keizer, ${ }^{54,47}$ M. Kelsey, ${ }^{67}$ M. Kenzie, ${ }^{55}$ T. Ketel, ${ }^{32}$ B. Khanji, ${ }^{47}$ A. Kharisova, ${ }^{82}$ S. Kholodenko, ${ }^{43}$ K. E. Kim, ${ }^{67}$ T. Kirn, ${ }^{13}$ V. S. Kirsebom, ${ }^{48}$ O. Kitouni, ${ }^{63}$ S. Klaver, ${ }^{31}$ K. Klimaszewski, ${ }^{35}$ S. Koliiev, ${ }^{51}$ A. Kondybayeva, ${ }^{80}$ A. Konoplyannikov, ${ }^{38}$ P. Kopciewicz, ${ }^{34}$ R. Kopecna, ${ }^{16}$ P. Koppenburg, ${ }^{31}$ M. Korolev, ${ }^{39}$ I. Kostiuk, ${ }^{31,51}$ O. Kot, ${ }^{51}$ S. Kotriakhova, ${ }^{37,30}$ P. Kravchenko, ${ }^{37}$ L. Kravchuk,${ }^{40}$ R. D. Krawczyk, ${ }^{47}$ M. Kreps,${ }^{55}$ F. Kress ${ }^{60}$ S. Kretzschmar, ${ }^{13}$ P. Krokovny, ${ }^{42, v}$ W. Krupa,${ }^{34}$ W. Krzemien, ${ }^{35}$ W. Kucewicz, ${ }^{86,33, k}$ M. Kucharczyk, ${ }^{33}$ V. Kudryavtsev, ${ }^{42, v}$ H. S. Kuindersma, ${ }^{31}$ G. J. Kunde, ${ }^{66}$ T. Kvaratskheliya,${ }^{38}$ D. Lacarrere, ${ }^{47}$ G. Lafferty, ${ }^{61}$ A. Lai, ${ }^{26}$ A. Lampis, ${ }^{26}$ D. Lancierini ${ }^{49}$ J. J. Lane,${ }^{61}$ R. Lane, ${ }^{53}$ G. Lanfranchi, ${ }^{22}$ C. Langenbruch, ${ }^{13}$ J. Langer, ${ }^{14}$ O. Lantwin, ${ }^{49,80}$ T. Latham, ${ }^{55}$ F. Lazzari, ${ }^{28, t}$ R. Le Gac, ${ }^{10}$ S. H. Lee, ${ }^{84}$ R. Lefèvre, ${ }^{9}$ A. Leflat,${ }^{39,47}$ S. Legotin, ${ }^{80}$ O. Leroy, ${ }^{10}$ T. Lesiak, ${ }^{33}$ B. Leverington, ${ }^{16} \mathrm{H} . \mathrm{Li},{ }^{71} \mathrm{~L} . \mathrm{Li},{ }^{62} \mathrm{P} . \mathrm{Li},{ }^{16}$ X. Li, ${ }^{66}$ Y. Li, ${ }^{6}$ Y. Li,${ }^{6}$ Z. Li,${ }^{67}$ X. Liang, ${ }^{67}$ T. Lin,${ }^{60}$ R. Lindner, ${ }^{47}$ V. Lisovskyi, ${ }^{14}$ R. Litvinov, ${ }^{26}$ G. Liu, ${ }^{71}$ H. Liu, ${ }^{5}$ S. Liu, ${ }^{6}$ X. Liu, ${ }^{3}$ A. Loi, ${ }^{26}$ J. Lomba Castro, ${ }^{45}$ I. Longstaff, ${ }^{58}$ J. H. Lopes, ${ }^{2}$ G. Loustau, ${ }^{49}$ G. H. Lovell, ${ }^{54}$ Y. Lu, ${ }^{6}$ D. Lucchesi, ${ }^{27, m}$ S. Luchuk, ${ }^{40}$ M. Lucio Martinez,${ }^{31}$ V. Lukashenko, ${ }^{31}$ Y. Luo, ${ }^{3}$ A. Lupato, ${ }^{61}$ E. Luppi, ${ }^{20, g}$ O. Lupton, ${ }^{55}$ A. Lusiani, ${ }^{28, r}$ X. Lyu, ${ }^{5}$ L. Ma, ${ }^{6}$ S. Maccolini, ${ }^{19, e}$ F. Machefert, ${ }^{11}$ F. Maciuc,${ }^{36}$ V. Macko, ${ }^{48}$ P. Mackowiak, ${ }^{14}$ S. Maddrell-Mander,${ }^{53}$ L. R. Madhan Mohan,${ }^{53}$ O. Maev,${ }^{37}$ A. Maevskiy, ${ }^{81}$ D. Maisuzenko, ${ }^{37}$ M. W. Majewski, ${ }^{34}$ S. Malde, ${ }^{62}$ B. Malecki, ${ }^{47}$ A. Malinin, ${ }^{79}$ T. Maltsev, ${ }^{42, v}$ H. Malygina, ${ }^{16}$ G. Manca, ${ }^{26, f}$ G. Mancinelli, ${ }^{10}$ R. Manera Escalero, ${ }^{44}$ D. Manuzzi, ${ }^{19, e}$ D. Marangotto, ${ }^{25,0}$ J. Maratas, ${ }^{9, u}$ J. F. Marchand, ${ }^{8}$ U. Marconi, ${ }^{19}$ S. Mariani,${ }^{21,47,21}$ C. Marin Benito, ${ }^{11}$ M. Marinangeli, ${ }^{48}$ P. Marino, ${ }^{48}$ J. Marks, ${ }^{16}$ P. J. Marshall,${ }^{59}$ G. Martellotti, ${ }^{30}$ L. Martinazzoli, ${ }^{47}$ M. Martinelli, ${ }^{24, i}$ D. Martinez Santos, ${ }^{45}$ F. Martinez Vidal ${ }^{46}$ A. Massafferri, ${ }^{1}$ M. Materok, ${ }^{13}$ R. Matev,${ }^{47}$ A. Mathad, ${ }^{49}$ Z. Mathe, ${ }^{47}$ V. Matiunin, ${ }^{38}$ C. Matteuzzi, ${ }^{24}$ K. R. Mattioli, ${ }^{84}$ A. Mauri, ${ }^{31}$ E. Maurice,${ }^{85,11, b}$ J. Mauricio, ${ }^{44}$ M. Mazurek, ${ }^{35}$ M. McCann, ${ }^{60}$ L. Mcconnell, ${ }^{17}$

T. H. Mcgrath, ${ }^{61}$ A. McNab, ${ }^{61}$ R. McNulty, ${ }^{17}$ J. V. Mead, ${ }^{59}$ B. Meadows,${ }^{64}$ C. Meaux, ${ }^{10}$ G. Meier,${ }^{14}$ N. Meinert,${ }^{75}$ D. Melnychuk, ${ }^{35}$ S. Meloni, ${ }^{24, \mathrm{i}}$ M. Merk,${ }^{31,78}$ A. Merli, ${ }^{25}$ L. Meyer Garcia, ${ }^{2}$ M. Mikhasenko, ${ }^{47}$ D. A. Milanes, ${ }^{73}$ E. Millard,${ }^{55}$ 
M.-N. Minard ${ }^{8}$ L. Minzoni, ${ }^{20, g}$ S. E. Mitchell,${ }^{57}$ B. Mitreska, ${ }^{61}$ D. S. Mitzel,${ }^{47}$ A. Mödden, ${ }^{14}$ R. A. Mohammed, ${ }^{62}$ R. D. Moise, ${ }^{60}$ T. Mombächer, ${ }^{14}$ I. A. Monroy, ${ }^{73}$ S. Monteil,${ }^{9}$ M. Morandin,${ }^{27}$ G. Morello, ${ }^{22}$ M. J. Morello, ${ }^{28, r}$ J. Moron, ${ }^{34}$ A. B. Morris ${ }^{74}$ A. G. Morris, ${ }^{55}$ R. Mountain, ${ }^{67}$ H. Mu, ${ }^{3}$ F. Muheim,${ }^{57}$ M. Mukherjee, ${ }^{7}$ M. Mulder, ${ }^{47}$ D. Müller, ${ }^{47}$ K. Müller, ${ }^{49}$ C. H. Murphy, ${ }^{62}$ D. Murray, ${ }^{61}$ P. Muzzetto, ${ }^{26}$ P. Naik ${ }^{53}$ T. Nakada,${ }^{48}$ R. Nandakumar, ${ }^{56}$ T. Nanut,${ }^{48}$ I. Nasteva, ${ }^{2}$ M. Needham ${ }^{57}$ I. Neri, ${ }^{20, \mathrm{~g}}$ N. Neri, ${ }^{25, \mathrm{o}}$ S. Neubert, ${ }^{74}$ N. Neufeld, ${ }^{47}$ R. Newcombe, ${ }^{60}$ T. D. Nguyen, ${ }^{48}$ C. Nguyen-Mau, ${ }^{48,1}$ E. M. Niel, ${ }^{11}$ S. Nieswand, ${ }^{13}$ N. Nikitin, ${ }^{39}$ N. S. Nolte, ${ }^{47}$ C. Nunez, ${ }^{84}$ A. Oblakowska-Mucha, ${ }^{34}$ V. Obraztsov, ${ }^{43}$ S. Ogilvy,${ }^{58}$ D. P. O'Hanlon, ${ }^{53}$ R. Oldeman, ${ }^{26, f}$ C. J. G. Onderwater, ${ }^{77}$ J. D. Osborn, ${ }^{84}$ A. Ossowska, ${ }^{33}$ J. M. Otalora Goicochea, ${ }^{2}$ T. Ovsiannikova, ${ }^{38}$ P. Owen, ${ }^{49}$ A. Oyanguren, ${ }^{46}$ B. Pagare,${ }^{55}$ P. R. Pais,${ }^{47}$ T. Pajero, ${ }^{28,47, \mathrm{r}}$ A. Palano, ${ }^{18}$ M. Palutan, ${ }^{22}$ Y. Pan,${ }^{61}$ G. Panshin, ${ }^{82}$ A. Papanestis, ${ }^{56}$ M. Pappagallo, ${ }^{57}$ L. L. Pappalardo, ${ }^{20, g}$ C. Pappenheimer, ${ }^{64}$ W. Parker ${ }^{65}$ C. Parkes, ${ }^{61}$ C. J. Parkinson, ${ }^{45}$ B. Passalacqua, ${ }^{20}$ G. Passaleva, ${ }^{21,47}$ A. Pastore, ${ }^{18}$ M. Patel,${ }^{60}$ C. Patrignani,,${ }^{19, e}$ C. J. Pawley, ${ }^{78}$ A. Pearce,${ }^{47}$ A. Pellegrino, ${ }^{31}$ M. Pepe Altarelli, ${ }^{47}$ S. Perazzini, ${ }^{19}$ D. Pereima, ${ }^{38}$ P. Perret,${ }^{9}$ K. Petridis, ${ }^{53}$ A. Petrolini, ${ }^{23, h}$ A. Petrov, ${ }^{79}$ S. Petrucci ${ }^{57}$ M. Petruzzo, ${ }^{25}$ A. Philippov, ${ }^{41}$ L. Pica, ${ }^{28}$ M. Piccini, ${ }^{76}$ B. Pietrzyk, ${ }^{8}$ G. Pietrzyk, ${ }^{48}$ M. Pili, ${ }^{62}$ D. Pinci, ${ }^{30}$ J. Pinzino, ${ }^{47}$ F. Pisani, ${ }^{47}$ A. Piucci,${ }^{16}$ Resmi P. K., ${ }^{10}$ V. Placinta, ${ }^{36}$ S. Playfer,${ }^{57}$ J. Plews, ${ }^{52}$ M. Plo Casasus, ${ }^{45}$ F. Polci, ${ }^{12}$ M. Poli Lener, ${ }^{22}$ M. Poliakova,${ }^{67}$ A. Poluektov, ${ }^{10}$ N. Polukhina, ${ }^{80, c}$ I. Polyakov, ${ }^{67}$ E. Polycarpo, ${ }^{2}$ G. J. Pomery, ${ }^{53}$ S. Ponce, ${ }^{47}$

A. Popov, ${ }^{43}$ D. Popov, ${ }^{5,47}$ S. Popov, ${ }^{41}$ S. Poslavskii, ${ }^{43}$ K. Prasanth,${ }^{33}$ L. Promberger, ${ }^{47}$ C. Prouve, ${ }^{45}$ V. Pugatch, ${ }^{51}$

A. Puig Navarro, ${ }^{49}$ H. Pullen, ${ }^{62}$ G. Punzi, ${ }^{28, n}$ W. Qian, ${ }^{5}$ J. Qin, ${ }^{5}$ R. Quagliani, ${ }^{12}$ B. Quintana, ${ }^{8}$ N. V. Raab, ${ }^{17}$

R. I. Rabadan Trejo, ${ }^{10}$ B. Rachwal, ${ }^{34}$ J. H. Rademacker ${ }^{53}$ M. Rama,${ }^{28}$ M. Ramos Pernas, ${ }^{45}$ M. S. Rangel, ${ }^{2}$ F. Ratnikov, ${ }^{41,81}$ G. Raven, ${ }^{32}$ M. Reboud, ${ }^{8}$ F. Redi, ${ }^{48}$ F. Reiss, ${ }^{12}$ C. Remon Alepuz, ${ }^{46}$ Z. Ren,${ }^{3}$ V. Renaudin, ${ }^{62}$ R. Ribatti, ${ }^{28}$ S. Ricciardi, ${ }^{56}$ D. S. Richards ${ }^{56}$ K. Rinnert, ${ }^{59}$ P. Robbe, ${ }^{11}$ A. Robert, ${ }^{12}$ G. Robertson, ${ }^{57}$ A. B. Rodrigues, ${ }^{48}$ E. Rodrigues, ${ }^{59}$

J. A. Rodriguez Lopez, ${ }^{73}$ M. Roehrken, ${ }^{47}$ A. Rollings, ${ }^{62}$ P. Roloff, ${ }^{47}$ V. Romanovskiy, ${ }^{43}$ M. Romero Lamas, ${ }^{45}$ A. Romero Vidal, ${ }^{45}$ J. D. Roth, ${ }^{84}$ M. Rotondo, ${ }^{22}$ M. S. Rudolph, ${ }^{67}$ T. Ruf, ${ }^{47}$ J. Ruiz Vidal, ${ }^{46}$ A. Ryzhikov, ${ }^{81}$ J. Ryzka, ${ }^{34}$ J. J. Saborido Silva, ${ }^{45}$ N. Sagidova, ${ }^{37}$ N. Sahoo,${ }^{55}$ B. Saitta, ${ }^{26, f}$ C. Sanchez Gras, ${ }^{31}$ C. Sanchez Mayordomo, ${ }^{46}$ R. Santacesaria, ${ }^{30}$ C. Santamarina Rios, ${ }^{45}$ M. Santimaria, ${ }^{22}$ E. Santovetti, ${ }^{29, j}$ D. Saranin, ${ }^{80}$ G. Sarpis, ${ }^{61}$ M. Sarpis,${ }^{74}$ A. Sarti, ${ }^{30}$ C. Satriano, ${ }^{30, q}$ A. Satta, ${ }^{29}$ M. Saur, ${ }^{5}$ D. Savrina, ${ }^{38,39}$ H. Sazak, ${ }^{9}$ L. G. Scantlebury Smead, ${ }^{62}$ S. Schael, ${ }^{13}$ M. Schellenberg, ${ }^{14}$ M. Schiller, ${ }^{58}$ H. Schindler, ${ }^{47}$ M. Schmelling, ${ }^{15}$ T. Schmelzer, ${ }^{14}$ B. Schmidt, ${ }^{47}$ O. Schneider, ${ }^{48}$ A. Schopper, ${ }^{47}$ M. Schubiger, ${ }^{31}$ S. Schulte, ${ }^{48}$ M. H. Schune, ${ }^{11}$ R. Schwemmer, ${ }^{47}$ B. Sciascia, ${ }^{22}$ A. Sciubba, ${ }^{30}$ S. Sellam, ${ }^{68}$ A. Semennikov, ${ }^{38}$ A. Sergi, ${ }^{52,47}$ N. Serra,${ }^{49}$ J. Serrano, ${ }^{10}$ L. Sestini, ${ }^{27}$ A. Seuthe, ${ }^{14}$ P. Seyfert, ${ }^{47}$ D. M. Shangase, ${ }^{84}$ M. Shapkin, ${ }^{43}$ I. Shchemerov ${ }^{80}$ L. Shchutska, ${ }^{48}$ T. Shears, ${ }^{59}$ L. Shekhtman, ${ }^{42, v}$ V. Shevchenko, ${ }^{79}$ E. B. Shields, ${ }^{24, i}$ E. Shmanin, ${ }^{80}$ J. D. Shupperd ${ }^{67}$ B. G. Siddi,${ }^{20}$ R. Silva Coutinho, ${ }^{49}$ G. Simi,${ }^{27}$ S. Simone, ${ }^{18, d}$ I. Skiba, ${ }^{20, g}$ N. Skidmore,${ }^{74}$ T. Skwarnicki, ${ }^{67}$ M. W. Slater, ${ }^{52}$ J. C. Smallwood, ${ }^{62}$ J. G. Smeaton, ${ }^{54}$ A. Smetkina, ${ }^{38}$ E. Smith, ${ }^{13}$ M. Smith, ${ }^{60}$ A. Snoch, ${ }^{31}$ M. Soares, ${ }^{19}$ L. Soares Lavra, ${ }^{9}$ M. D. Sokoloff, ${ }^{64}$ F. J. P. Soler ${ }^{58}$ A. Solovev, ${ }^{37}$ I. Solovyev, ${ }^{37}$ F. L. Souza De Almeida, ${ }^{2}$ B. Souza De Paula, ${ }^{2}$ B. Spaan, ${ }^{14}$ E. Spadaro Norella, ${ }^{25,0}$ P. Spradlin, ${ }^{58}$ F. Stagni, ${ }^{47}$ M. Stahl, ${ }^{64}$ S. Stahl, ${ }^{47}$ P. Stefko, ${ }^{48}$ O. Steinkamp, ${ }^{49,80}$ S. Stemmle, ${ }^{16}$ O. Stenyakin, ${ }^{43}$ H. Stevens, ${ }^{14}$ S. Stone,${ }^{67}$ M. E. Stramaglia, ${ }^{48}$ M. Straticiuc, ${ }^{36}$ D. Strekalina, ${ }^{80}$ S. Strokov, ${ }^{82}$ F. Suljik, ${ }^{62}$ J. Sun, ${ }^{26}$ L. Sun, ${ }^{72}$ Y. Sun, ${ }^{65}$ P. Svihra,${ }^{61}$ P. N. Swallow, ${ }^{52}$ K. Swientek, ${ }^{34}$ A. Szabelski, ${ }^{35}$ T. Szumlak,${ }^{34}$ M. Szymanski, ${ }^{47}$ S. Taneja, ${ }^{61}$ Z. Tang, ${ }^{3}$ T. Tekampe,${ }^{14}$ F. Teubert, ${ }^{47}$ E. Thomas, ${ }^{47}$ K. A. Thomson, ${ }^{59}$ M. J. Tilley, ${ }^{60}$ V. Tisserand, ${ }^{9}$ S. T' Jampens, ${ }^{8}$ M. Tobin, ${ }^{6}$ S. Tolk, ${ }^{47}$ L. Tomassetti, ${ }^{20, g}$ D. Torres Machado, ${ }^{1}$ D. Y. Tou,${ }^{12}$ M. Traill,${ }^{58}$ M. T. Tran,${ }^{48}$ E. Trifonova,${ }^{80}$ C. Trippl, ${ }^{48}$ A. Tsaregorodtsev,${ }^{10}$ G. Tuci, ${ }^{28, n}$ A. Tully,${ }^{48}$ N. Tuning,${ }^{31}$ A. Ukleja, ${ }^{35}$ D. J. Unverzagt, ${ }^{16}$ A. Usachov, ${ }^{31}$ A. Ustyuzhanin, ${ }^{41,81}$ U. Uwer ${ }^{16}$ A. Vagner, ${ }^{82}$ V. Vagnoni, ${ }^{19}$ A. Valassi ${ }^{47}$ G. Valenti, ${ }^{19}$ M. van Beuzekom, ${ }^{31}$ H. Van Hecke, ${ }^{66}$ E. van Herwijnen, ${ }^{80}$ C. B. Van Hulse, ${ }^{17}$ M. van Veghel, ${ }^{77}$ R. Vazquez Gomez, ${ }^{45}$ P. Vazquez Regueiro, ${ }^{45}$ C. Vázquez Sierra ${ }^{31}$ S. Vecchi,${ }^{20}$ J. J. Velthuis, ${ }^{53}$ M. Veltri, ${ }^{21, p}$ A. Venkateswaran, ${ }^{67}$ M. Veronesi, ${ }^{31}$ M. Vesterinen, ${ }^{55}$ D. Vieira ${ }^{64}$ M. Vieites Diaz,${ }^{48}$ H. Viemann, ${ }^{75}$ X. Vilasis-Cardona, ${ }^{83}$ E. Vilella Figueras, ${ }^{59}$ P. Vincent, ${ }^{12}$ G. Vitali, ${ }^{28}$ A. Vitkovskiy, ${ }^{31}$ A. Vollhardt, ${ }^{49}$ D. Vom Bruch, ${ }^{12}$ A. Vorobyev, ${ }^{37}$ V. Vorobyev, ${ }^{42, v}$ N. Voropaev, ${ }^{37}$ R. Waldi, ${ }^{75}$ J. Walsh, ${ }^{28}$ C. Wang, ${ }^{16}$ J. Wang, ${ }^{3}$ J. Wang, ${ }^{72}$ J. Wang, ${ }^{4}$ J. Wang, ${ }^{6}$ M. Wang, ${ }^{3}$ R. Wang, ${ }^{53}$ Y. Wang, ${ }^{7}$ Z. Wang, ${ }^{49}$ D. R. Ward, ${ }^{54}$ H. M. Wark, ${ }^{59}$ N. K. Watson, ${ }^{52}$ S. G. Weber, ${ }^{12}$ D. Websdale, ${ }^{60}$ C. Weisser,${ }^{63}$ B. D. C. Westhenry, ${ }^{53}$ D. J. White, ${ }^{61}$ M. Whitehead ${ }^{53}$ D. Wiedner, ${ }^{14}$ G. Wilkinson, ${ }^{62}$ M. Wilkinson, ${ }^{67}$ I. Williams, ${ }^{54}$ M. Williams, ${ }^{63,69}$ M. R. J. Williams, ${ }^{61}$ F. F. Wilson, ${ }^{56}$ W. Wislicki, ${ }^{35}$ M. Witek,${ }^{33}$ L. Witola, ${ }^{16}$ G. Wormser, ${ }^{11}$ S. A. Wotton, ${ }^{54}$ H. Wu, ${ }^{67}$ K. Wyllie, ${ }^{47}$ Z. Xiang, ${ }^{5}$ D. Xiao, ${ }^{7}$ Y. Xie, ${ }^{7}$ H. Xing, ${ }^{71}$ A. Xu, ${ }^{4}$ J. Xu, ${ }^{5}$ L. Xu, ${ }^{3}$ M. Xu, ${ }^{7}$ Q. Xu,,${ }^{5}$ Z. Xu, ${ }^{5}$ Z. Xu, ${ }^{4}$ D. Yang, ${ }^{3}$ Y. Yang, ${ }^{5}$ Z. Yang, ${ }^{3}$ Z. Yang, ${ }^{65}$ Y. Yao, ${ }^{67}$ L. E. Yeomans, ${ }^{59}$ H. Yin, ${ }^{7}$ J. Yu, ${ }^{7}$ X. Yuan, ${ }^{67}$ O. Yushchenko, ${ }^{43}$ K. A. Zarebski, ${ }^{52}$ M. Zavertyaev, ${ }^{15, \mathrm{c}}$ M. Zdybal, ${ }^{33}$ O. Zenaiev, ${ }^{47}$ M. Zeng, ${ }^{3}$ D. Zhang, ${ }^{7}$ L. Zhang, ${ }^{3}$ S. Zhang, ${ }^{4}$ Y. Zhang, ${ }^{47}$ 
 A. Zhelezov, ${ }^{16}$ Y. Zheng, ${ }^{5}$ X. Zhou, ${ }^{5}$ Y. Zhou, ${ }^{5}$ X. Zhu, ${ }^{3}$ V. Zhukov, ${ }^{13,39}$ J. B. Zonneveld, ${ }^{57}$ S. Zucchelli, ${ }^{19, e}$
D. Zuliani, ${ }^{27}$ and G. Zunica ${ }^{61}$

(LHCb Collaboration)

\author{
${ }^{1}$ Centro Brasileiro de Pesquisas Físicas (CBPF), Rio de Janeiro, Brazil \\ ${ }^{2}$ Universidade Federal do Rio de Janeiro (UFRJ), Rio de Janeiro, Brazil \\ ${ }^{3}$ Center for High Energy Physics, Tsinghua University, Beijing, China \\ ${ }^{4}$ School of Physics State Key Laboratory of Nuclear Physics and Technology, Peking University, \\ Beijing, China \\ ${ }^{5}$ University of Chinese Academy of Sciences, Beijing, China \\ ${ }^{6}$ Institute Of High Energy Physics (IHEP), Beijing, China \\ ${ }^{7}$ Institute of Particle Physics, Central China Normal University, Wuhan, Hubei, China \\ ${ }^{8}$ Université Grenoble Alpes, Université Savoie Mont Blanc, CNRS, IN2P3-LAPP, Annecy, France \\ ${ }^{9}$ Université Clermont Auvergne, CNRS/IN2P3, LPC, Clermont-Ferrand, France \\ ${ }^{10}$ Aix Marseille Université, CNRS/IN2P3, CPPM, Marseille, France \\ ${ }^{11}$ Université Paris-Saclay, CNRS/IN2P3, IJCLab, Orsay, France \\ ${ }^{12}$ LPNHE, Sorbonne Université, Paris Diderot Sorbonne Paris Cité, CNRS/IN2P3, Paris, France \\ ${ }^{13}$ I. Physikalisches Institut, RWTH Aachen University, Aachen, Germany \\ ${ }^{14}$ Fakultät Physik, Technische Universität Dortmund, Dortmund, Germany \\ ${ }^{15}$ Max-Planck-Institut für Kernphysik (MPIK), Heidelberg, Germany \\ ${ }^{16}$ Physikalisches Institut, Ruprecht-Karls-Universität Heidelberg, Heidelberg, Germany \\ ${ }^{17}$ School of Physics, University College Dublin, Dublin, Ireland \\ ${ }^{18}$ INFN Sezione di Bari, Bari, Italy \\ ${ }^{19}$ INFN Sezione di Bologna, Bologna, Italy \\ ${ }^{20}$ INFN Sezione di Ferrara, Ferrara, Italy \\ ${ }^{21}$ INFN Sezione di Firenze, Firenze, Italy \\ ${ }^{22}$ INFN Laboratori Nazionali di Frascati, Frascati, Italy \\ ${ }^{23}$ INFN Sezione di Genova, Genova, Italy \\ ${ }^{24}$ INFN Sezione di Milano-Bicocca, Milano, Italy \\ ${ }^{25}$ INFN Sezione di Milano, Milano, Italy \\ ${ }^{26}$ INFN Sezione di Cagliari, Monserrato, Italy \\ ${ }^{27}$ Universita degli Studi di Padova, Universita e INFN, Padova, Padova, Italy \\ ${ }^{28}$ INFN Sezione di Pisa, Pisa, Italy \\ ${ }^{29}$ INFN Sezione di Roma Tor Vergata, Roma, Italy \\ ${ }^{30}$ INFN Sezione di Roma La Sapienza, Roma, Italy \\ ${ }^{31}$ Nikhef National Institute for Subatomic Physics, Amsterdam, Netherlands \\ ${ }^{32}$ Nikhef National Institute for Subatomic Physics and VU University Amsterdam, Amsterdam, Netherlands \\ ${ }^{33}$ Henryk Niewodniczanski Institute of Nuclear Physics Polish Academy of Sciences, Kraków, Poland \\ ${ }^{34}$ AGH-University of Science and Technology, Faculty of Physics and Applied Computer Science, \\ Kraków, Poland \\ ${ }^{35}$ National Center for Nuclear Research (NCBJ), Warsaw, Poland \\ ${ }^{36}$ Horia Hulubei National Institute of Physics and Nuclear Engineering, Bucharest-Magurele, Romania \\ ${ }^{37}$ Petersburg Nuclear Physics Institute NRC Kurchatov Institute (PNPI NRC KI), Gatchina, Russia \\ ${ }^{38}$ Institute of Theoretical and Experimental Physics NRC Kurchatov Institute (ITEP NRC KI), \\ Moscow, Russia, Moscow, Russia \\ ${ }^{39}$ Institute of Nuclear Physics, Moscow State University (SINP MSU), Moscow, Russia \\ ${ }^{40}$ Institute for Nuclear Research of the Russian Academy of Sciences (INR RAS), Moscow, Russia \\ ${ }^{41}$ Yandex School of Data Analysis, Moscow, Russia \\ ${ }^{42}$ Budker Institute of Nuclear Physics (SB RAS), Novosibirsk, Russia \\ ${ }^{43}$ Institute for High Energy Physics NRC Kurchatov Institute (IHEP NRC KI), \\ Protvino, Russia, Protvino, Russia \\ ${ }^{44}$ ICCUB, Universitat de Barcelona, Barcelona, Spain \\ ${ }^{45}$ Instituto Galego de Física de Altas Enerxías (IGFAE), Universidade de Santiago de Compostela, \\ Santiago de Compostela, Spain \\ ${ }^{46}$ Instituto de Fisica Corpuscular, Centro Mixto Universidad de Valencia-CSIC, Valencia, Spain \\ ${ }^{47}$ European Organization for Nuclear Research (CERN), Geneva, Switzerland \\ ${ }^{48}$ Institute of Physics, Ecole Polytechnique Fédérale de Lausanne (EPFL), Lausanne, Switzerland \\ ${ }^{49}$ Physik-Institut, Universität Zürich, Zürich, Switzerland
}


${ }^{50}$ NSC Kharkiv Institute of Physics and Technology (NSC KIPT), Kharkiv, Ukraine

${ }^{51}$ Institute for Nuclear Research of the National Academy of Sciences (KINR), Kyiv, Ukraine

${ }^{52}$ University of Birmingham, Birmingham, United Kingdom

${ }^{53}$ H.H. Wills Physics Laboratory, University of Bristol, Bristol, United Kingdom

${ }^{54}$ Cavendish Laboratory, University of Cambridge, Cambridge, United Kingdom

${ }^{55}$ Department of Physics, University of Warwick, Coventry, United Kingdom

${ }^{56}$ STFC Rutherford Appleton Laboratory, Didcot, United Kingdom

${ }^{57}$ School of Physics and Astronomy, University of Edinburgh, Edinburgh, United Kingdom

${ }^{58}$ School of Physics and Astronomy, University of Glasgow, Glasgow, United Kingdom

${ }^{59}$ Oliver Lodge Laboratory, University of Liverpool, Liverpool, United Kingdom

${ }^{60}$ Imperial College London, London, United Kingdom

${ }^{61}$ Department of Physics and Astronomy, University of Manchester, Manchester, United Kingdom

${ }^{62}$ Department of Physics, University of Oxford, Oxford, United Kingdom

${ }^{63}$ Massachusetts Institute of Technology, Cambridge, Massachusetts, USA

${ }^{64}$ University of Cincinnati, Cincinnati, Ohio, USA

${ }^{65}$ University of Maryland, College Park, Maryland, USA

${ }^{66}$ Los Alamos National Laboratory (LANL), Los Alamos, New Mexico, USA

${ }^{67}$ Syracuse University, Syracuse, New York, USA

${ }^{68}$ Laboratory of Mathematical and Subatomic Physics, Constantine, Algeria [associated with Universidade Federal do Rio de Janeiro (UFRJ), Rio de Janeiro, Brazil]

${ }^{69}$ School of Physics and Astronomy, Monash University, Melbourne, Australia (associated with Department of Physics, University of Warwick, Coventry, United Kingdom)

${ }^{70}$ Pontifícia Universidade Católica do Rio de Janeiro (PUC-Rio), Rio de Janeiro, Brazil [associated with Universidade Federal do Rio de Janeiro (UFRJ), Rio de Janeiro, Brazil]

${ }^{71}$ Guangdong Provencial Key Laboratory of Nuclear Science, Institute of Quantum Matter, South China Normal University, Guangzhou, China (associated with Center for High Energy Physics, Tsinghua University, Beijing, China)

${ }^{72}$ School of Physics and Technology, Wuhan University, Wuhan, China (associated with Center for High Energy Physics, Tsinghua University, Beijing, China)

${ }^{73}$ Departamento de Fisica, Universidad Nacional de Colombia, Bogota, Colombia (associated with LPNHE, Sorbonne Université, Paris Diderot Sorbonne Paris Cité, CNRS/IN2P3, Paris, France)

${ }^{74}$ Universität Bonn-Helmholtz-Institut für Strahlen und Kernphysik, Bonn, Germany (associated with Physikalisches Institut, Ruprecht-Karls-Universität Heidelberg, Heidelberg, Germany)

${ }^{75}$ Institut für Physik, Universität Rostock, Rostock, Germany (associated with Physikalisches Institut, Ruprecht-Karls-Universität Heidelberg, Heidelberg, Germany)

${ }^{76}$ INFN Sezione di Perugia, Perugia, Italy (associated with INFN Sezione di Ferrara, Ferrara, Italy)

${ }^{77}$ Van Swinderen Institute, University of Groningen, Groningen, Netherlands (associated with Nikhef National Institute for Subatomic Physics, Amsterdam, Netherlands)

${ }^{78}$ Universiteit Maastricht, Maastricht, Netherlands (associated with Nikhef National Institute for Subatomic Physics, Amsterdam, Netherlands)

${ }^{79}$ National Research Centre Kurchatov Institute, Moscow, Russia [associated with Institute of Theoretical and Experimental Physics NRC Kurchatov Institute (ITEP NRC KI), Moscow, Russia]

${ }^{80}$ National University of Science and Technology "MISIS", Moscow, Russia [associated with Institute of Theoretical and Experimental Physics NRC Kurchatov Institute (ITEP NRC KI), Moscow, Russia]

${ }^{81}$ National Research University Higher School of Economics, Moscow, Russia (associated with Yandex School of Data Analysis, Moscow, Russia)

${ }^{82}$ National Research Tomsk Polytechnic University, Tomsk, Russia [associated with Institute of Theoretical and Experimental Physics NRC Kurchatov Institute (ITEP NRC KI), Moscow, Russia]

${ }^{83}$ DS4DS, La Salle, Universitat Ramon Llull, Barcelona, Spain (associated with ICCUB, Universitat de Barcelona, Barcelona, Spain)

${ }^{84}$ University of Michigan, Ann Arbor, Michigan, USA (associated with Syracuse University, Syracuse, New York, USA)

${ }^{85}$ Laboratoire Leprince-Ringuet, Palaiseau, France

${ }^{86}$ AGH-University of Science and Technology, Faculty of Computer Science, Electronics and Telecommunications, Kraków, Poland

${ }^{a}$ Also at Universidade Federal do Triângulo Mineiro (UFTM), Uberaba-MG, Brazil.

${ }^{\mathrm{b}}$ Also at Laboratoire Leprince-Ringuet, Palaiseau, France.

${ }^{c}$ Also at P.N. Lebedev Physical Institute, Russian Academy of Science (LPI RAS), Moscow, Russia.

Also at Università di Bari, Bari, Italy. 
${ }^{\mathrm{e}}$ Also at Università di Bologna, Bologna, Italy.

${ }^{\mathrm{f}}$ Also at Università di Cagliari, Cagliari, Italy.

${ }^{g}$ Also at Università di Ferrara, Ferrara, Italy.

${ }^{\mathrm{h}}$ Also at Università di Genova, Genova, Italy.

${ }^{\mathrm{i}}$ Also at Università di Milano Bicocca, Milano, Italy.

${ }^{\mathrm{j}}$ Also at Università di Roma Tor Vergata, Roma, Italy.

${ }^{k}$ Also at AGH - University of Science and Technology, Faculty of Computer Science, Electronics and Telecommunications, Kraków, Poland.

${ }^{1}$ Also at Hanoi University of Science, Hanoi, Vietnam.

m Also at Università di Padova, Padova, Italy.

${ }^{\mathrm{n}}$ Also at Università di Pisa, Pisa, Italy.

${ }^{\circ}$ Also at Università degli Studi di Milano, Milano, Italy.

${ }^{\mathrm{p}}$ Also at Università di Urbino, Urbino, Italy.

${ }^{\mathrm{q}}$ Also at Università della Basilicata, Potenza, Italy.

${ }^{\mathrm{r}}$ Also at Scuola Normale Superiore, Pisa, Italy.

${ }^{\mathrm{s}}$ Also at Università di Modena e Reggio Emilia, Modena, Italy.

${ }^{\mathrm{t}}$ Also at Università di Siena, Siena, Italy.

${ }^{\mathrm{u}}$ Also at MSU - Iligan Institute of Technology (MSU-IIT), Iligan, Philippines.

${ }^{v}$ Also at Novosibirsk State University, Novosibirsk, Russia.

${ }^{\mathrm{w}}$ Also at INFN Sezione di Trieste, Trieste, Italy. 